Methods We evaluated 1771 adolescents at 13 year old as part of a population-based cohort study (EPITeen). Sleep duration was estimated by the difference between self-reported usual bedtimes and wake-up times and adolescents were classified in three categories: $\leq 8.5 \mathrm{~h}$ (reference class), $>8.5 \mathrm{~h}$ and $<9.5 \mathrm{~h}$ and $\geq 9.5 \mathrm{~h}$. Blood pressure (BP) was measured with a mercury sphygmomanometer using the auscultatory method, and hypertension was defined according to the American Academy of Pediatrics criteria. To evaluate the association between BP and sleep duration, OR and respective $95 \% \mathrm{CI}$, were computed, using the binary regression models adjusted for parents' education, BMI and caffeine intake.

Results The mean (SD) sleep duration was 9.04 (0.80) hours per day. The prevalence of hypertension was $22.4 \%$ and it was significantly higher among males (54.8\% vs $45.2 \%$; $=0.001)$. After adjustment, in females, a positive association was found between sleep duration and hypertension $(>8.5 \mathrm{~h}$ and $<9.5 \mathrm{~h}: \mathrm{OR}=1.61,95 \% \mathrm{CI} 1.07$ to 2.44 ; $\geq 9.5$ h: $\mathrm{OR}=1.75$, 95\% CI 1.13 to 2.70$)$. Among males an inverse association was found, significant only in those who slept $\geq 9.5 \mathrm{~h}(\mathrm{OR}=0.62,95 \% \mathrm{CI} 0.40$ to 0.95$)$.

Conclusion Sleep duration was positively associated with the odds of hypertension occurrence in females, but the opposite association was found in males.

\section{P2-234 SELF-REPORTED HEARING LOSS AND USE OF HEALTH SERVICES FOR ELDERLY: A POPULATION BASED STUDY IN SãO PAULO CITY, BRAZIL}

doi:10.1136/jech.2011.142976j.67

K M Paiva, ${ }^{*} N$ Farias, C L G Cesar, M B de Azevedo Barros, L Carandina, M C G P Alves, M Goldbaum. University of São Paulo, São Paulo, Brazil

Introduction Hearing loss has been identified as one of the most frequent chronic conditions affecting elderly people and can be result in social isolation, depression and increased dependency. The use of health services to preventive ends it is important to evaluate the health behaviours of elderly with disabilities.

Methods Data are from the Survey of Health of São Paulo (ISACapital), a population-based cross-sectional study $(n=3357)$. We analysed the subgroup of elderly (60 years and above $-n=872$ ) to determine the association between reported hearing loss and use of health services: immunisation against influenza in last year, participation in prevention programs: prostate cancer, breast cancer and cervical cancer. We used the $\chi^{2}$ test of association and analysis of Poisson regression (significance level: 0.05).

Results The prevalence of hearing loss in elderly people was $11.2 \%$ and it was higher in men than women $(p=0.00) .60 .5 \%$ of the elderly related to take immunisation against influenza, $82.9 \%$ of the women referred to participate the program of prevention of cervical cancer and $64.1 \%$ referred to participate the breast cancer one. There was an association between self-reported hearing loss and participate in programs to prevent prostate cancer (RP: 1,$8 ; p=0.03$ ).

Conclusion Self-reported hearing loss can be itself a revelation indicator of handicap and it is quick and inexpensive to be performed. These data should be a tool to evaluate of the use of health services and moreover they could help to plan of hearing rehabilitation services.

\section{P2-235 VERBAL FLUENCY TESTS RELIABILITY IN A BRAZILIAN MULTICENTRIC STUDY, ELSA BRASIL}

doi:10.1136/jech.2011.142976j.68

${ }^{1} V$ Passos, ${ }^{*}{ }^{1} \mathrm{~L}$ Giatti, ${ }^{1} \mathrm{~S}$ Barreto, ${ }^{1} \mathrm{R}$ Figueiredo, ${ }^{1} \mathrm{P}$ Caramelli, ${ }^{2} \mathrm{~B}$ Bensenor, ${ }^{3} \mathrm{M}$ de Jesus Fonseca, ${ }^{4} \mathrm{~N}$ Cade, ${ }^{2} \mathrm{~A}$ Goulart, ${ }^{5} \mathrm{M}$ A Nunes, ${ }^{3,4} \mathrm{M}$ Alves, ${ }^{6} \mathrm{~A}$ A Trindade. ${ }^{1}$ Faculty of Medicine, Federal University of Minas Gerais, Belo Horizonte, Minas Gerais, Brazil; ${ }^{2}$ São Paulo University, São Paulo, São Paulo, Brazil; ${ }^{3}$ Oswaldo Cruz Foundation, Rio de
Janeiro, Rio de Janeiro, Brazil; ${ }^{4}$ Federal University of Espirito Santo, Vitória, Espirito Santo, Brazil; ${ }^{5}$ Federal University of Rio Grande do Sul, Porto Alegre, Rio Grande do Sul, Brazil; ${ }^{6}$ Federal University of Bahia, Salvador, Bahia, Brazil

Introduction Because of the pronounced and fast population ageing of low and middle-income countries, dementia is now a leading cause of disability. Verbal Fluency Tests (VFT) were used to assess cognitive functions in ELSA (Longitudinal Study of Adults' Health Brasil), a cohort that investigates incidence and predictors of chronic diseases among 15000 civil servants (35-74 years old). The performance in VFT needs to be evaluated by a trained investigator, in order to count the words excluding repetitions and intrusions. As multicentric studies have to concern about the homogeneity of their interpretation, this study investigates the reliability of VFT scoring by supervisors of the six ELSA research centers, who independently judged 120 category (animals) and 120 phonemic (F letter) tests.

Methods The scores were compared to a reference standard score obtained by independent judgement of two experts and ratings reliability obtained by Intraclass Correlation Coefficient (ICC) and Bland-Altman plot.

Results Scores were very similar among centers and a high level of agreement was observed between each center and the reference standard. ICC values for both tests varied from 0.979 (95\% CI 0.970 to 0.985 ) to 0.987 (95\% CI 0.981 to 0.991 ). The Bland-Altman plot showed that the mean difference is small for both tests, and $95 \%$ of scores are located between the mean and 2 SDs.

Conclusion These results show the importance of the implemented measures of quality assurance and control and allow ELSA to proceed upon its main objective, to identify social, psychological and biological predictors of cognitive decline in a Brazilian adult population.

\section{P2-236 BETTER COGNITIVE FUNCTION ASSOCIATED WITH WHO RECOMMENDED FRUIT AND VEGETABLE INTAKE IN A LOW- INCOME ELDERLY POPULATION IN BRAZIL}

\author{
doi:10.1136/jech.2011.142976j.69
}

${ }^{1} \mathrm{M}$ Pastor-Valero, ${ }^{*} \mathrm{R}$ Furlan-Viebig, ${ }^{2} \mathrm{P}$ R Menezes, ${ }^{3} \mathrm{M}$ Scazufca. ${ }^{1}$ Departamento de Salud Pública, Universidad Miguel Hernández, Sant Joan d'Alacant, Spain; ${ }^{2}$ Departamento de Medicina Preventiva, Faculdade de Medicina, Universidade de São Paulo, São Paulo, Brazil; ${ }^{3}$ Instituto e Departamento de Psiquiatria, Faculdade de Medicina, Universidade de São Paulo, São Paulo, Brazil

Globally, cognitive deficit and dementia are among the major agerelated chronic disorders. Daily consumption of fruit and vegetables has been associated with a diminished risk of cognitive deficit later in life. We examined whether the WHO recommendations of a daily intake of five servings of fruit and vegetables ( $\geq 400 \mathrm{gm}$ ) were associated with cognition in a low-income elderly population in the city of São Paulo. We explored the influence of socio-demographic characteristics, lifestyle factors and health measures on this association. Methods We performed a cross-sectional analyses with 1849 participants $\geq 65$ years old, free of dementia, at the baseline of the population-based prospective SPAH study. Cognition function was assessed using the Community Screening Instrument for Dementia (CSI-D), (scored as $0-30$ ). Few cases with scores $=0$ were excluded. Cognitive deficit was defined as scores $\leq 1.5 \mathrm{SDs}$ of the mean population (CSI-D scores $\leq 20.93$ ). Fruit and vegetable intake was estimated using a Food Frequency Questionnaire. The association between cognition and fruit and vegetable intake was investigated using multiple linear and logistic regression models.

Results Multivariate analyses showed that a higher intake of fruit and vegetables was associated with higher cognitive scores. Individuals who consumed $\geq 400 \mathrm{~g}$ /day presented a significant $42 \%$ lower risk of cognitive deficit. Nevertheless, adjusting for education significantly attenuated this association. 